\title{
Materials Science and Engineering at the National Laboratories A Perspective from Industry R.A. Laudise
}

The DOE-funded multipurpose National Laboratories have annual budgets in excess of $\$ 3$ billion and a substantial fraction of their work is devoted to materials research and engineering. Indeed, in the aggregate, the National Laboratories together consume a significant fraction of U.S. Department of Energy monies spent annually on materials science. In terms of staff and equipment, they are a national treasure.

The defense-oriented National Laboratories have provided the materials understanding, the new materials required for demanding environments, and the extreme reliability essential for U.S. nuclear credibility. Their response to their past mission and the need to continue this mission in materials is, or ought to be, unquestioned.

\section{...the raison d'etre of the National Laboratories is not to generate production technology for industry.}

On the other hand, as emphasized in the Packard Report, ${ }^{*}$ it became clear the original missions for many of the other laboratories-to establish the scientific and technical basis for nuclear, and later-on alternative, energy sources-were often no longer a national priority. Hence, materials work in support of these activities was not always the best allocation of resources.

In the past five years much has been done to re-focus missions, taking into account the strengths of the various laborato-

${ }^{*}$ Report of the White House Science Council, Federal Laboratory Review Panel, Office of Science and Technology, May 1983. ries. The newly emerging missions have significant materials science and engineering content. However, the excellent quality of the human resources and the magnitude of the financial resources expended should motivate continued efforts to improve mission focus.

The words of the Packard Report are still applicable:

"...the clearer a laboratory's missions are, the better the performance will be....missions must be consistent with the laboratory's existing strengths and expertise ....missions of the Federal laboratories [should] be defined to encourage cooperation rather than competition with industry and universities...."

With the above in mind, it is appropriate to ask two questions: What should National Laboratory staff and management do to ensure the success and survival of their materials activities? What can materials scientist outside the laboratories do to help? The following is more an agenda for discussion than any claim to pat answers.

\section{What Can the National Laboratories Do?}

...Realize that funding is largely a zero sum game...that resource refocusing rather than enlargement is the name of the game.

While it is important to improve chances that the science produced in the National Laboratories leads to useful technology and to set up mechanisms to assure appropriate technology transfer...the raison d'etre of the National Laboratories is not to generate production technology for industry. Setting this as a principal goal is programming for failure.

Experience shows that technology is most effective when generated close to its point of use and under conditions where institutional barriers to transfer are minimal. True, we should work harder to ex- tract technological value from the National Laboratories' science, and it will be necessary for the laboratories to modify their internal structures when appropriate. However, it is essential to remember that in terms of the National Laboratories, useful technology is only a small amount of icing on a much larger scientific cake; science is where we should focus our major attention for mission improvement.

The proper activity for each National Laboratory is to contribute the highest quality science to the information base pertinent to its mission. A particular laboratory's mission and the part of the knowledge base to be enlarged should be chosen to be vital to nationally prioritized technological directions.

Appropriate technology activities should be carried on in the National Laboratories to assure relevant communication with putative customers and to decrease the impedance mismatch between science and engineering applications.

\section{...science is where we should focus our major attention for mission improvement.}

That part of the knowledge base chosen for enhancement should be an area where present university and private sector industrial research is unable or unlikely to provide the needed information.

This is all easy to say, but picking a particular area and mission will be difficult. The choices will be crucial. They ought to result not from shooting at targets of opportunity, not from following a particular fad, but from balanced considered studies involving questions such as the following few examples: What does the semiconductor industry need that the National Laboratories might provide? What are the lacunae in our understanding of superconductivity, high strength ceramics, light alloys, special performance polymers, etc.? How can processing be made a science? Which nationally relevant materials areas need more rigor?

It will be necessary to make difficult choices and encourage-no, forcespecialization at various laboratories. But doing nearly everything is not an acceptable choice.

\section{What Can Outsiders Do To Help?}

..Play an active role in the National Laboratories' search for missions...realize that 
decisions are crucial because they will employ a national treasure and present a unique opportunity to focus our scientific powers on key materials problems...encourage in every way the exchange of people, ideas and priority views between the National Laboratories, the private sector and universities.

While it might be argued that it is primarily the job of National Laboratory personnel to be pro-activist in seeking contacts, sabbaticals, exchanges, crossinstitutional seminars, and personnel transfers, it is essential for the survival of materials science in industry and academia that U.S. science and technology and its

\section{Not drawing on the resources of the National Laboratories is competing with a voluntarily assumed handicap.}

materials industry remain first-class world competitive. Not drawing on the resources of the National Laboratories is competing with a voluntarily assumed handicap.

The National Laboratories need help ... and so do the rest of $u$ s. What National
Laboratory could help you the most? What would you like them to do? Do they know this? Is there a National Laboratory intern in your organization, or a cooperative research program, or a jointly supervised thesis? When did you last visit a National Laboratory?

Some of the best materials science in the world is done in our National Laboratories. Let's boost it and help it in its efforts, well under way, to have impact. The opportunities are too great and the penalties too severe for "business as usual" in materials activities at the National Laboratories.

R.A. Laudise is a materials researcher and a manager of industrial research.

\section{Pressure Measurementand Control}

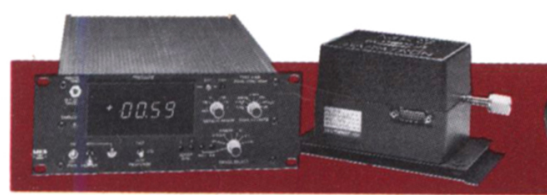

Type 270B and Type 390/398 High Accuracy Baratron $^{\otimes}$ System

Temperature controlled precisely at $45^{\circ} \mathrm{C}$ for superior zero and span stability. Accuracy $0.08 \%$ of Reading, optional $0.05 \%$.

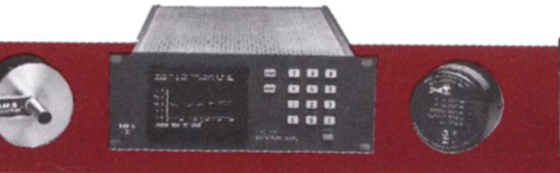

Type 122/127/112A - Pressure Programmer/ Display and 100 Series Baratron Sensors Computer based instrument, with application specific software to measure, display, analyze and contról process parameters for pressure,

vacuum, and flow.

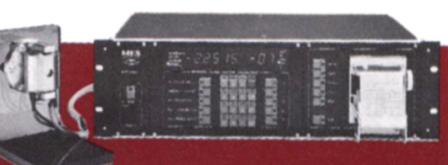

Spinning Rotor Friction Gauge The transfer standard for vacuum gauge calibration in the range of $10^{-7}$ to $10^{-2}$ torr with accuracy of $1 \%$ of Reading $\pm 3 \times 10^{-8}$ torr.

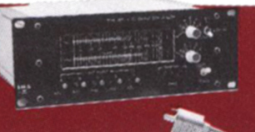

Type 286/287 Thermocouple Vacuum Gauges

Economical vacuum process pressure measurements from 2 to $10^{-2}$ torr in non-corrosive environments. 2 channel and 5 channel.
High vacuum pressure measurement system with Charge-Rate* circuitry to allow continuous ranging from $10^{-3}$ to $10^{-9}$ torr.

Our complete line of pressure measurement and control instruments represent the ultimate in electronic manometer simplicity, economy and performance.
'U.S. Patent No. 4,0355,720 Foreign Patents and Patents Pending

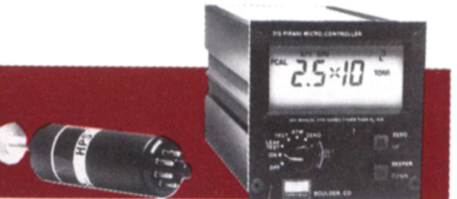

HPS Series 315 Pirani Gauge System Continuous high performance general purpose vacuum measurement from $10^{-3}$ to 100 torr with discrete points to atmosphere. Gauge tube features temperature compensation for improved Pirani Gauge stability. 


\section{The Environmental Scanning Electron Microscope...}

\section{Seeing Things As They Really Are}

Enter a new dimension in electron microscopy, and see the real world in real time. ElectroScan's ESEM, the world's first Environmental Scanning Electron Microscope, allows direct observation of specimens in their natural states, as they go through physical and chemical changes.

Analyze samples as you've never seen them before in an ordinary SEM-wet specimens, insulators and uncoated specimens. Witness dynamic processes-wetting, drying, absorption, melting, corrosion and crystallization.

The unique capabilities of the ESEM-continuous pressure control of its chamber environment and ElectroScan's exclusive secondary electron detector-have eliminated the need for sample preparation and infinitely expanded the range of EM applications.

The ESEM is the only way of viewing specimens as they really are...the new laboratory equipment standard for solving virtually any EM application problem with speed and simplicity.

For your demonstration of the ESEM, contact ElectroScan now.

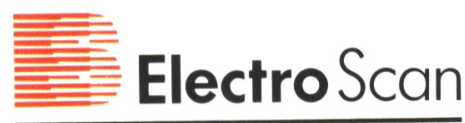

ElectroScan Corporation 100 Rosewood Drive Danvers, MA 01923 (508) 777-9280 